\title{
Commentary on "Novel Insights from Clinical Practice p.G360R Is a Pathogenic GLA Gene Mutation Responsible for a Classic Phenotype of Fabry Disease"
}

\author{
Walter Serra \\ Cardiology Unit, Azienda Ospedaliero Universitaria di Parma, Parma, Italy
}

In this original paper, "Novel Insights from Clinical Practice p.G360R is a pathogenic GLA gene mutation responsible for a classic phenotype of Fabry disease," Carvalho Silva et al. [1] report the case of a classic phenotype of Fabry disease in a 60-year-old male patient presenting with left ventricular hypertrophy and stroke. Genetic analysis revealed two GLA gene variants - the previously described variant p.R356Q (currently considered as benign or associated with milder disease) and the novel p.G360R mutation (predicted as probably pathogenic) - probably disrupting the dimerization interface of the protein and therefore causing the classic phenotype. The patient underwent cardiac magnetic resonance and echocardiography that confirmed severe and concentric left ventricular hypertrophy, although with thin apical walls, and revealed late gadolinium enhancement.

The mutation of the GLA gene p.Gly360Arg (c.1078G >C) was deposited in Clinical Variation in 2016 by a single clinical center, clinically tested but with uncertain significance, but the pathogenicity of the mutation could now be confirmed by this article.

As reported in the authors' conclusions, "this case also warns about the importance of the awareness on Fabry disease red flags and the appropriate diagnosis of this

\section{KARGER}

(c) 2019 S. Karger AG, Basel

E-Mail karger@karger.com

www.karger.com/crd condition, given the potential prognostic benefit that could result from timely and adequate specific therapy."

Anderson-Fabry disease (MAF) is a lysosomal accumulation due to molecular defects in the GLA (alpha-galactosidase) gene causing the progressive buildup of intracellular GB3. The disease is dominant X-linked, so in addition to malignant males, almost $100 \%$ of female carriers exhibit phenotypic signs after the third/fourth decade [2]. Patients with MAF may be sent for cardiology evaluation after clinical diagnosis of the disease or may accidentally arrive at the presence of typical clinical signs of the disease, but entirely unspecific, such as palpitations, arrhythmias, and dyspnea due to altered myocardial perfusion under stress.

Among the late signs, we find, in both sexes, angina attacks due to the accumulation of endothelial and muscle cells in intramyocardial vessels. Gb3 buildup within endothelial cells and smooth arterial musculature results in a complete response to vasomotor stimuli, creating anatomical and functional damage and, consequently, an alteration of myocardial perfusion. Subsequently, major complications such as myocardial ischemia, ventricular arrhythmias, and congestive heart failure are achieved [3,4]. Suspicion may arise with the cardiologist by combining the presence of signs and symptoms and taking into account 
Fig. 1. 2D speckle-tracking echocardiography shows a reduced strain and systolic longitudinal SR and reduced early diastolic SR in patients with Fabry disease.

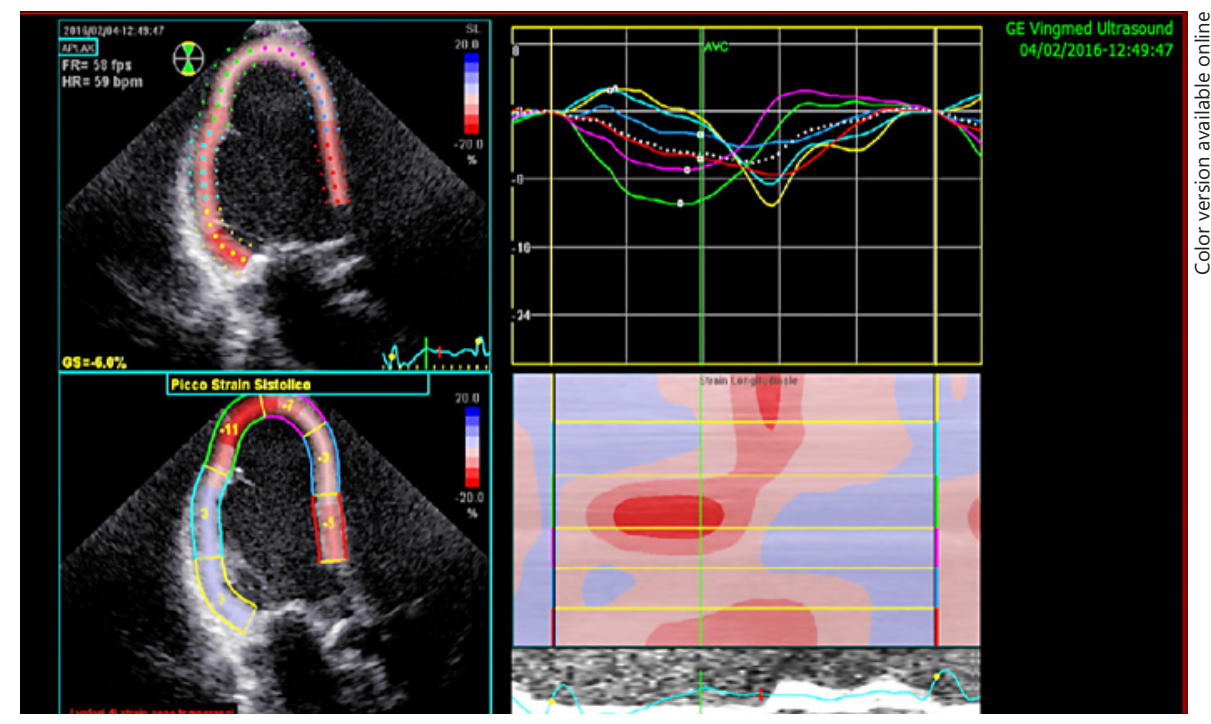

the involvement of different organs of the body. Nowadays, a failure to diagnose MAF involves ethical and legal problems because there is effective and specific therapy approved by major regulatory agencies (FDA and EMEA).

Many accumulation diseases are associated with left ventricular cardiac hypertrophy. Some of these are linked to chromosome X. MAF is the most common form of lysosomal accumulation of cardiac involvement in adults, with a prevalence of $0.5-1 \%$ in patients with hypertrophic cardiomyopathy [5].

It is necessary to follow a diagnostic algorithm in order to make a precise diagnosis in the so-called "phenocopies" of hypertrophic cardiomyopathy, such as hemochromatosis, sarcoidosis, and amyloidosis. A diagnostic algorithm includes ECG, ECG Holter $24 \mathrm{~h}$, echocardiography, and BNP (every 12-24 months); CPET, echocardiography, and MRI (within 5 years of the first assessment); as well as coronary angiography, and myocardial biopsy in cases of uncertain etiology. An important biomarker is lyso-Gb3, a degradation product of Gb3. It has been shown that the plasma concentration of lyso-Gb3 is high in all classic Fabry patients. Furthermore, it is slightly elevated in late-onset atypical mutations. Overall, the lyso-Gb3 level is considered to predict the potential severity of unknown mutations.

\section{Imaging}

At the myocardial level, the accumulation of Gb3/GL3 renders the cardiomyocytes hypertrophied, with consequent triggering of fibrosis and thickening of the ventric- ular walls. Echocardiography highlights the mechanisms of secondary hypertrophy that leads to an increase in the thickness of the interventricular septum and the left posterior ventricular wall. The diagnosis of cardiac involvement can be obtained with echocardiography by DTI analysis: the isovolumic relaxation time is longer (IVRT), the isovolumic contraction time is shorter (IVCT), and the peak speed of movement of the systolic wall is lower (SA) [6, 7]. 2D speckle-tracking echocardiography shows a reduced strain and systolic longitudinal strain rate (SR) and reduced early diastolic SR in patients with Fabry disease (Fig. 1). The extent of cardiomyocyte hypertrophy and the accumulation of glycosphingolipids within the vacuoles correlate with the extension of the thickening of the left ventricle wall, visible to magnetic resonance with gadolinium $[8,9]$. Heart valves are involved in the disease and, typically, the thickening of the aortic and mitral valve leaflets may be present in a percentage of cases in both children (20\%) and adults. Cardiac magnetic resonance provides important information about tissue characterization using gadolinium and allows aspects of differential diagnosis between various hypertrophic cardiomyopathies. In particular, a lower relaxation time (T2) seems to be typical compared to patients with hypertrophic sarcomere cardiomyopathy and the relief of hyperenhancement at the level of the inferior-lateral wall of the left ventricle, with the introduction of the MOLLI (modified look-locker inversion recovery imaging) strategy. This new technique revealed reduced T1 mapping and an increased extracellular volume in Fabry cardiomyopathy $[9,10]$. 


\section{References}

1 Carvalho Silva D, Marques N, Azevedo O, Mittenberger Miltenyi G, et al. Novel Insights from Clinical Practice p.G360R is a pathogenic GLA gene mutation responsible for a classic phenotype of Fabry disease. Cardiology. 2019, DOI: $10.1159 / 000502437$.

2 Garman SC, Garboczi DN. The molecular defect leading to Fabry disease: structure of human a-galactosidase. J Mol Biol. 2004 Mar; 337(2):319-35.

3 Weidemann F, Linhart A, Monserrat L, Strotmann LJ. Cardiac challenges in patients with Fabry disease. Int J Cardiol. 2010 May;141(1): 3-10.

4 Pieroni M, Chimenti C, De Cobelli F, Morgante E, Del Maschio A, Gaudio C, et al. Fabry's disease cardiomyopathy: echocardiographic detection of endomyocardial glycosphingolipid compartmentalization. J Am Coll Cardiol. 2006 Apr;47(8):1663-71.
5 Elliott PM, Anastasakis A, Borger MA, Borggrefe M, Cecchi F, Charron P, et al. 2014 ESC Guidelines on diagnosis and management of hypertrophic cardiomyopathy: the Task Force for the Diagnosis and Management of Hypertrophic Cardiomyopathy of the European Society of Cardiology (ESC). Eur Heart J. 2014 Oct;35(39):2733-79.

6 Linhart A, Kampmann C, Zamorano JL, Sunder-Plassmann G, Beck M, Mehta A, et al.; European FOS Investigators. Cardiac manifestations of Anderson-Fabry disease: results from the international Fabry outcome survey. Eur Heart J. 2007 May;28(10):1228-35.

7 Zamorano J, Serra V, Pérez de Isla L, Feltes G, Calli A, Barbado FJ, et al. Usefulness of tissue Doppler on early detection of cardiac disease in Fabry patients and potential role of enzyme replacement therapy (ERT) for avoiding progression of disease. Eur J Echocardiogr. 2011 Sep;12(9):671-7.
8 Moon JC, Sachdev B, Elkington AG, McKenna WJ, Mehta A, Pennell DJ, et al. Gadolinium enhanced cardiovascular magnetic resonance in Anderson-Fabry disease. Evidence for a disease specific abnormality of the myocardial interstitium. Eur Heart J. 2003 Dec; 24(23):2151-5.

9 Radenkovic D, Weingärtner S, Ricketts L, Moon JC, Captur G. T1 mapping in cardiac MRI. Heart Fail Rev. 2017 Jul;22(4):415-30.

10 Nordin S, Kozor R, Bulluck H, Castelletti S, Rosmini S, Abdel-Gadir A, et al. Cardiac Fabry Disease With Late Gadolinium Enhancement Is a Chronic Inflammatory Cardiomyopathy. J Am Coll Cardiol. 2016 Oct;68(15): $1707-8$ 